\title{
Non-linear response of a liquid bridge to a sinusoidal acceleration under microgravity
}

\author{
I. Martínez, J.M. Perales, J. Meseguer
}

\begin{abstract}
An experiment was performed aboard a sounding rocket on a long cylindrical liquid bridge, aiming at discerning the real transfer function of this liquid configuration to small acceleration loads, quantified by the liquid free-surface deformation divided by the axially imposed acceleration. The results were, however, in great discrepancy with theoretical predictions, showing asymmetric jumps of high amplitude in the evolution of the radial deformation of the liquid bridge, instead of a symmetric sinusoidal radial deformation (axisymmetry was preserved). It has been found now that a non-linear dynamic model perfectly explains this unexpected behaviour.
\end{abstract}

\section{1}

\section{Introduction}

A liquid bridge is a simplified model of more complex fluid configurations, as in the floating zone technique of crystal growth, and consists of an isothermal mass of liquid with constant properties (density $\rho$, surface tension $\sigma$ and kinematic viscosity $v$ ) held by surface tension forces between two parallel and coaxial circular disks of the same radii $R$, placed at a distance $L$ apart, as sketched in Fig. 1 . The liquid bridge interface, whose deformation is the subject of this research, is anchored to both disks, with the two contact lines solid-liquid-air being circumferences pinned to the edges of the disks. An axial sinusoidally varying acceleration of the discs $g$ (along the axis of symmetry of the liquid column) constitutes the excitation applied.

Free-surface equilibrium shapes and stability limits of the liquid bridge configuration are defined by the following dimensionless parameters: the slenderness $\Lambda=L /(2 R)$, the dimensionless volume of liquid $V=V^{\star} /\left(\pi \mathrm{R}^{2} L\right)$, where $V^{*}$ stands for the physical volume, and the axial Bond number $B=\rho \mathrm{gR}^{2} / \sigma$ (neglecting the density of the sur-

We acknowledge the support we have had on data retrieval and ancillary information from the Texus team and the financial support from the Spanish Ministerio de Ciencia y Tecnología. rounding media). For a weightless right circular-cylinder liquid bridge, the well-known Rayleigh stability limit holds: the liquid column becomes unstable when its length is larger than its circumference $(\Lambda>\pi)$, and beyond this limit the liquid column breaks in two drops by asymmetric necking.

The response of a liquid bridge to several mechanical stimuli has been theoretically predicted and experimentally verified in terrestrial laboratories with small millimetric bridges and with larger bridges in a Plateautank configuration (surrounding the bridge by an immiscible liquid of nearly the same density), as well as on space platforms. However, in some space experiments, puzzling results were obtained, attributable to the unavoidable mechanically noisy ambient of a very light and very active manned orbiting vehicle (Martínez et al. 1995).

To clarify those unexplained results, the experiment here studied was executed (aboard sounding rocket Texus33), aiming at obtaining a precise record of the interface deformation caused by the axial acceleration imposed, to check it with available analytical models: linear and static, with corrections for non-linear and for dynamic effects (Martínez et al. 1996).

However, in this experiment, because of unforeseen circumstances, important dynamic effects were excited, and the liquid bridge started to oscillate in an unexpected way, not satisfactorily explained in a previous work (Martínez et al. 1996). The experiment is revisited in this paper, and the experimental dynamic behaviour is analysed by using a one-dimensional model for the liquid bridge dynamics, based on the Cosserat model already used in liquid bridge dynamics, which retains most dynamics and non-linear effects at a much lower cost than full three-dimensional models.

The study of non-linear dynamics and breakage of liquid bridges was started more than 20 years ago, initially using an inviscid, one-dimensional slices model. This initial model was developed from one used in capillary jet studies, just modifying the boundary condition (Meseguer 1983; Rivas and Meseguer 1984; Meseguer and Sanz 1985). Sirignano and Mehring (2000) have recently published a wide review of the theories commonly used in liquid jet breaking, and an historical review of the most representative publications concerning the breaking of liquid bridges can be found in Zhang et al. (1996). These reviews should be completed with some recent papers (Padday et al. 1997; Yildirim and Basaran 2001, Montanero 2003), which are mostly devoted to the breaking of stretching liquid bridges. It has to be stressed that one-dimensional 


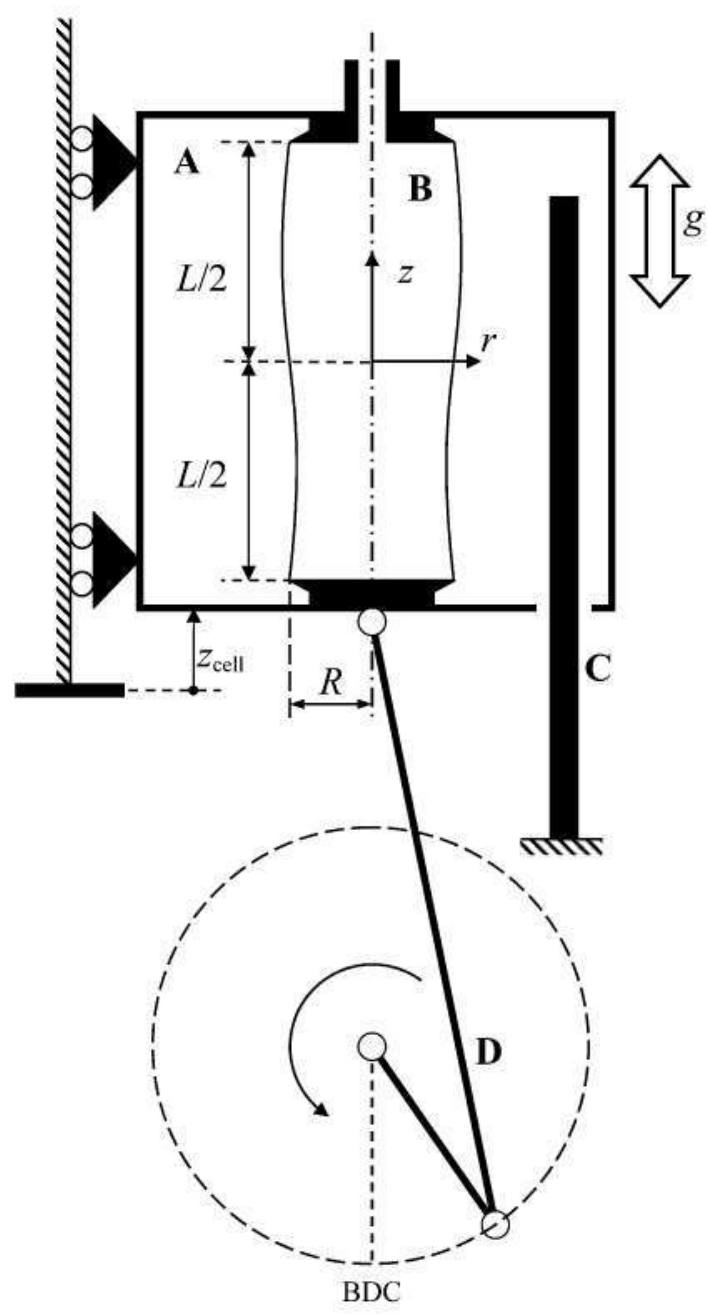

Fig. 1. Sketch of a liquid bridge in air, held between two connected solid discs being oscillated. Oscillations start at bottom dead centre (BDC). A liquid bridge cell; B liquid bridge; $\mathrm{C}$ fix rod; D crank-shaft mechanism. Viewing CCD-camera moves with the cell

models are suitable for explaining the liquid bridge dynamics provided the liquid bridge slenderness is high enough, as happens in this case where it is $\Lambda>2.83$.

\section{2}

\section{Experiment description}

The goal of this experiment was to check the static response of the liquid column acting as an accelerometer, by subjecting it to a calibrated microgravity acceleration $\left(100 \pm 1 \mu \mathrm{g}\right.$, i.e. $\left.10^{-3} \mathrm{~m} / \mathrm{s}^{2}\right)$. This stimuli is well above the expected uncontrolled g-jitters of the Texus platform (known to be below $10 \mu \mathrm{g}$ from previous rocket flights and measured also during this experiment), but well below the expected breakage by capillary instability of the liquid bridge selected, at some $200 \mu \mathrm{g}$ (Martínez et al. 1996).

A picture of the hardware used in the experiment is shown in Fig. 2; it consists of the following main elements (see also the sketch in Fig. 1):

- A liquid bridge cell, where a long $(L=85 \mathrm{~mm})$ column of silicone oil (AK-10, Wacher Chemie, Munich, Germany, with density $\rho=920 \mathrm{~kg} / \mathrm{m}^{3}$, kinematic viscosity $v=10 \cdot 10^{-6} \mathrm{~m}^{2} / \mathrm{s}$ and oil-air interface tension

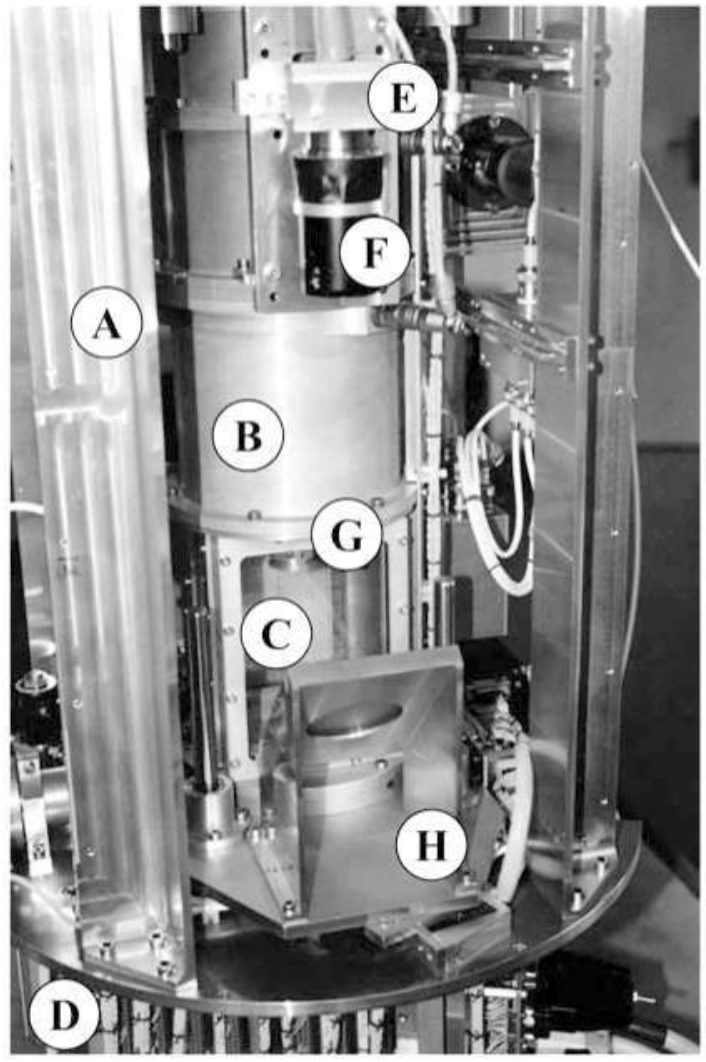

Fig. 2. Experimental equipment (Texus 33 TEM-09 liquid bridge cell). A rocket module structure; $\mathrm{B}$ liquid reservoir; $\mathrm{C}$ liquid bridge cell (without liquid); D electronics box; E CCD camera; $\mathrm{F}$ lens; $\mathrm{G}$ upper disc; $\mathrm{H}$ mirror at $45^{\circ}$

$\sigma=0.02 \mathrm{~N} / \mathrm{m}$ ), is established between two equal coaxial metal discs ( $R=15 \mathrm{~mm}$ in radius), to the edge of which the liquid is naturally anchored. The liquid volume injection mechanism takes care to adjust the injected volume to that corresponding to a circular cylinder of the same length and same radius.

- A liquid bridge visualisation set-up with a CCD camera, a mirror and a background diffuse illumination, including a raster for reference in the pictures.

- A liquid bridge module, fixed to the sounding rocket, inside which the liquid bridge cell as a whole (including discs, reservoir, mirror and camera) is made to oscillate axially along the axis of the rocket (coincident with the axis of the liquid bridge), by means of the crank-shaft mechanism depicted in Fig. 1.

The experimental sequence, described in more detail in Martínez et al. (1996), is:

1. Formation of the liquid bridge during the first minute after the sounding rocket attains microgravity (ballistic motion without air drag).

2. Idle period of $20 \mathrm{~s}$ to observe the initial decay of disturbances owing to the filling process; although it was known that the half damping time is $t_{1 / 2} \approx 35 \pm 5 \mathrm{~s}$, a shorter period was chosen because this decay period is followed by another transient event (the start of oscillations) that requires further decay time.

3. Five oscillation cycles of $45 \mathrm{~s}$ period each. 
4. Idle period of the remaining 1 minute of rocket flight, to look at the decay, before rocket re-entry and fall.

The response of the liquid bridge to small accelerations can be fully characterised by its deformation amplitude because all deformed shapes approach sinusoidal shapes (the first eigenmode). As the experiment aimed at measuring very small accelerations, the sensitivity had to be very large (the slenderness $\Lambda$ has to be near the Plateau-Rayleigh stability limit of $\Lambda=\pi$ ), but it is well-known that very sensitive systems are also very unstable and a compromise must be reached $(\Lambda=2.83$, corresponding to $\Lambda / \pi=0.9$, was selected for the experiment).

The crank-shaft mechanism implemented, produced a sinusoidal displacement of the liquid cell, $z_{\text {cell }}(t)$ (see Fig. 1), with the following kinematics:

$z_{\text {cell }}(t)=A_{1}\left(1-\cos \frac{2 \pi t}{T}\right)-A_{2}\left(1-\cos \frac{4 \pi t}{T}\right)$,

with measured values from the hardware (a linear potentiometer between rocket and fluid cell) of

$A_{1}=49.8 \pm 0.2 \mathrm{~mm}, A_{2}=4.1 \pm 0.2 \mathrm{~mm}$ and $T=45.2 \pm 0.2 \mathrm{~s}$. Notice that the crank-shaft mechanism only adds even harmonics $\left(A_{2}, A_{4}, A_{6}, \ldots\right)$ to the first one $\left(A_{1}\right)$. In our case, from crank-shaft kinematics, the neglected $(1-\cos (8 \pi t / T)$ ) term in (1) has a theoretical value of $A_{4}=0.03 \mathrm{~mm}$ that fell below the design resolution.

Fluid-cell acceleration, relative to the rocket (that can be assumed an inertial reference frame because of its relative mass and the overall ballistic trajectory), is also redundantly measured from the liquid bridge images, since a solid rod fixed to the rocket module is in the field of view (to the travelling camera, it is as if the rod were moving up and down instead of the liquid bridge cell; see the sketch in Fig. 1). The matching of the two-term crank-shaft motion law (1) deduced from potentiometer records with the actual rod displacement and acceleration measured from the images is perfect ( $\pm 0.1 \mathrm{~mm}$ in amplitude and $\pm 0.1 \mathrm{~s}$ in time), thus the acceleration applied to the liquid in liquid-cell axes is:

$g(t)=\frac{\mathrm{d}^{2} z_{\text {cell }}(t)}{\mathrm{d} t^{2}}=a_{1} \cos \frac{2 \pi t}{T}-a_{2} \cos \frac{4 \pi t}{T}$,

with $a_{1}=A_{1}(2 \pi / T)^{2}=0.96 \pm 0.02 \mathrm{~mm} / \mathrm{s}^{2}, a_{2}=A_{2}(4 \pi /$ $T)^{2}=0.32 \pm 0.02 \mathrm{~mm} / \mathrm{s}^{2}$ and $T=45.2 \pm 0.2 \mathrm{~s}$, or, in nondimensional terms:

$B(t)=\frac{\rho R^{2}}{\sigma} \frac{\mathrm{d}^{2} z_{\text {cell }}(t)}{\mathrm{d} t^{2}}=B_{1} \cos \frac{2 \pi t}{T}-B_{2} \cos \frac{4 \pi t}{T}$,

with $B_{1}=0.010 \pm 0.001$ and $B_{2}=0.0033 \pm 0.0003$.

\section{3}

\section{Analytical background}

To compare the theoretical predictions with the experimental results, a modal analysis is done, in order to find a first approximation to the oscillating shape,

$\frac{R(z, t)}{R}=1+a(t) \sin \frac{\pi z}{\Lambda}$, i.e. we aim at finding the liquid deformation amplitude, $a(t)$, coincident with the radial deformation at one quarter of the liquid column length $R(\Lambda / 2, t) / R-1=a(t)$, in terms of the non-dimensional acceleration applied, $B(t)$. A non-linear quasi-static analysis, as described in Martínez et al. (1996), could not fully explain the observed behaviour of the liquid, and dynamic models had to be used.

But neither the linear dynamic spring-type simulation developed in Martínez et al. (1996), namely:

$\frac{\mathrm{d}^{2} a(t)}{\mathrm{d} t^{2}}+\frac{2 \ln 2}{t_{1 / 2}} \frac{\mathrm{d} a(t)}{\mathrm{d} t}+\left(\frac{2 \pi}{T}\right)^{2} a(t)=K B(t)$

( $t_{1 / 2}$ being the half-time for viscous decay known from previous experiments with the same geometry and fluid, $T$ the forcing period and $K$ a known constant from the static analysis), gave satisfactory results Trying to elucidate possible hardware uncertainties, in Martínez et al. (1996), an additional third harmonic in the displacement imposed (1) was introduced in the load term $B(t)$ of (5) with a value of $A_{3}=0.25 \mathrm{~mm}$ empirically found by best fitting the actual liquid deformation, as shown in Martínez et al. (1996). This artificial input could not satisfactory be attributed to any conceivable load either intentionally applied or caused by platform noise.

Fortunately, an improved analytical model has been used (Eq. 6) that perfectly predicts the real response, showing that the third harmonic experimentally found in the radial amplitude, does not come from the load $B(t)$ in (6), but from the non-linear dynamic response of the liquid to the actual two-harmonic excitation.

The theoretical model that really matches the observed behaviour is, after Meseguer et al. (1994):

$m \frac{\mathrm{d}^{2} a(t)}{\mathrm{d} t^{2}}+m C \frac{\mathrm{d} a(t)}{\mathrm{d} t}+\lambda a(t)+\frac{3}{4} a(t)^{3}=B(t)$,

with $m=25 / 8$ being an analytical non-dimensional inertia-term coefficient (deduced considering the simplified inner velocity field given by the so-called Cosserat model $), C=O h^{1 / 2}=v(\rho /(\sigma \mathrm{R}))^{1 / 2}=0.0175$ the square root of the Ohnesorge number accounting for viscous dissipation (with $v=10 \cdot 10^{-6} \mathrm{~m}^{2} / \mathrm{s}, \rho=920 \mathrm{~kg} / \mathrm{m}^{3}$, $\sigma=0.020 \mathrm{~N} / \mathrm{m}, R=0.015 \mathrm{~m}$ ), with $\hat{\lambda}=\Lambda / \pi-1$ being a normalised slenderness having a small value and $B(t)$ being the non-dimensional forcing acceleration as before. The actual values from the two last coefficients $(\Lambda / \pi-1$ and 3/4) can be deduced just by making an analysis of the static equilibrium of the liquid bridge retaining not only the first but the following non-zero term). Equation 6 is a Duffing's equation, typical of soft springs (the cubic term softens the linear rigidity, introducing the non-linear effects).

4

Results

The results presented here come from the digitisation of the on-board recorded VHS video tape, to a $320 \times 240$ pixel map of 16 bit grey-scale, in a sequence of 3,600 images, with a $0.1 \mathrm{~s}$ time-span (the original video has 30 frames 


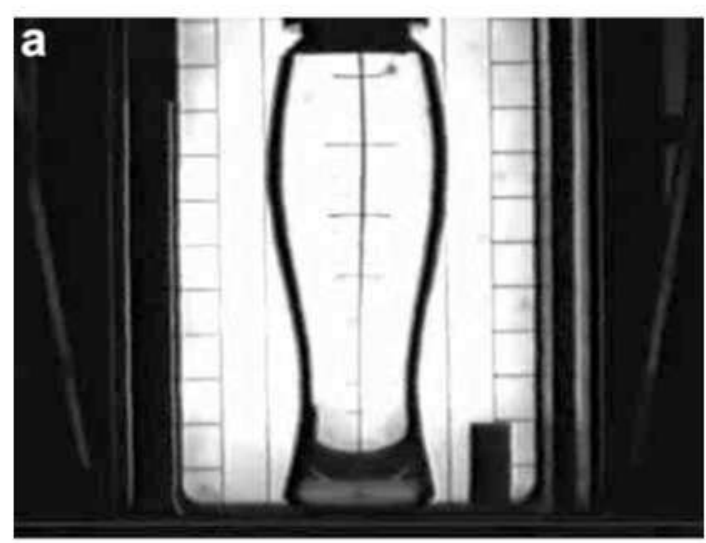

b

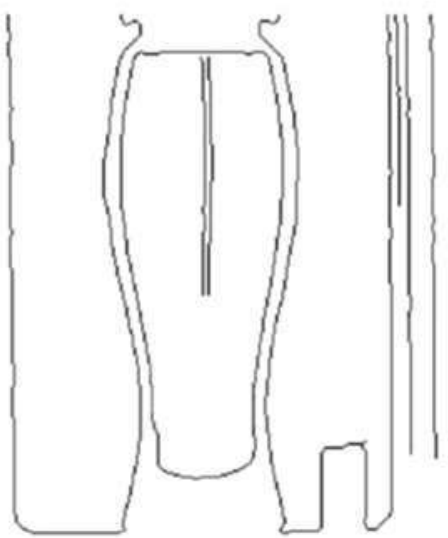

Fig. 3. a An actual image of the liquid bridge (amphora-shape outline over a white background). $\mathrm{b}$ Automatic edge extraction

per second), and analysed with Matlab software (The MathWorks Inc., Natick, MA, USA), that has been used to make all the computations and plots from the image sequence; in particular, the edges of the liquid bridge are automatically extracted using the Canny algorithm, which finds edges by looking for local maxima of the gradient of the grey-level matrix, using the derivative of a Gaussian filter. An actual image and its outline (automatic edge detection) are presented in Fig. 3. It might be noticed that the liquid column and the raster appear at a different scale; this is because of the conical perspective (looking from $420 \mathrm{~mm}$ far to an $86 \mathrm{~mm}$ liquid column length at the object plane, the raster being $48 \mathrm{~mm}$ further behind).

As mentioned above, because of the sinusoidal shape deformation (4), the response of the liquid bridge can be fully characterised by the deformation amplitude at one quarter of its length $(z=\Lambda / 2), a(t)=R(\Lambda / 2, t) / R-1$, but, to minimise uncertainty, instead of just this one radius measure local fitting, as performed in Martínez et al. (1996), here $a(t)$ is computed by fitting the whole outer shape in each image, $R(z, t)$, by a least-square technique explained below.

To measure the departure (as a function of time) of the liquid volume relative to a perfect cylinder, $v=V^{\star} /\left(\pi \mathrm{R}^{2} L\right)-1$, expected to be nil, one may just measure the radial departure at mid-length and apply a known linear theoretical relation (Martínez et al. 1996); $R(0, t) /$ $R=1+0.92 v(t)$ for $\Lambda=2.83$. But again, the uncertainty is smaller (goes down from 1 to $0.1 \%$ ) if we make the integration of all liquid discretised slices and compute the volume deviation by:

$v(t)=\frac{\sum R^{2}\left(z_{i}, t\right) \Delta z_{i}}{R^{2} L}-1$,

the result being shown in Fig. 4. Similarly, amphora-type amplitude deformations, i.e. $a(t)$ in (4), may be computed from a local measure of $R(\Lambda / 2, t) / R-1$, but the uncertainty is of $1 \%$ and a better fit is obtained by minimising the shape distance, i.e. $\Sigma\left(R(z, t)-R\left(z_{i}, t\right)\right)^{2}=\min$, that yields:

$a(t)=\frac{\sum R\left(z_{i}, t\right) \sin \frac{\pi z_{i}}{\Lambda} \Delta z_{i}}{R L}$,

with the result shown in Fig. 4. The lateral non-axisymmetric (comb-like) deformation, $Y(z, t)$, of the liquid bridge midway between the discs, normalised with the disc radius, $Y(0, t) / R$ (not sketched in Fig. 1), is also presented in Fig. 4, to give an idea of how well axial symmetry was preserved during the experiment and to show a small cross-coupling with the forcing frequency applied to the cell.

From Fig. 4, one can see a constant volume process (with a $0.3 \%$ uncertainty in volume), a similar behaviour for the combing (centrifugal bending) of the centre line (but with an unexplained $0.4 \%$ amplitude waving at the excitation frequency, maybe caused by some imperfection in the applied stimuli), and an amphora-type radial deformation $a(t)$ in (4), evolving with the following characteristics:

1. The transients (both, those coming from the injection manoeuvre and those coming from the abrupt start of the oscillation) disappear after the first three cycles as expected.

2. After oscillation suddenly stops, unexpectedly the transients are almost non-existent. This is a most remarkable fact and contrary to expectations, but successfully explained below.

3. The time-averaged shape deformation amplitude during the whole microgravity period in this experiment corresponds to an equivalent steady-state Bond number smaller than $1 \mu \mathrm{g}$, in contrast with averaged values measured on Spacelab experiments $(70 \mu \mathrm{g}$ in Spacelab-1 and $5 \mu \mathrm{g}$ in Spacelab-D1). It might be the platform- and mission-dependent effect.

The results of the non-linear dynamic simulation (6), with the two-term excitations (2), are presented in Fig. 5 in comparison with the experimental results, i.e. the non-dimensional stimuli $B(t)$, the experimental response $a(t)$ (dots), the non-linear dynamic simulation response (heavy solid line) and the linear dynamic simulation response (thin solid line). It can be seen that the linear dynamic simulation does not reproduce qualitatively all features of the experimental results, whereas the non-linear simulation does. Quantitatively, the non-linear simulation agrees with experimental results everywhere except in the final free dumped oscillations where a readjustment in the initial conditions of the simulation would lead to a better match. 

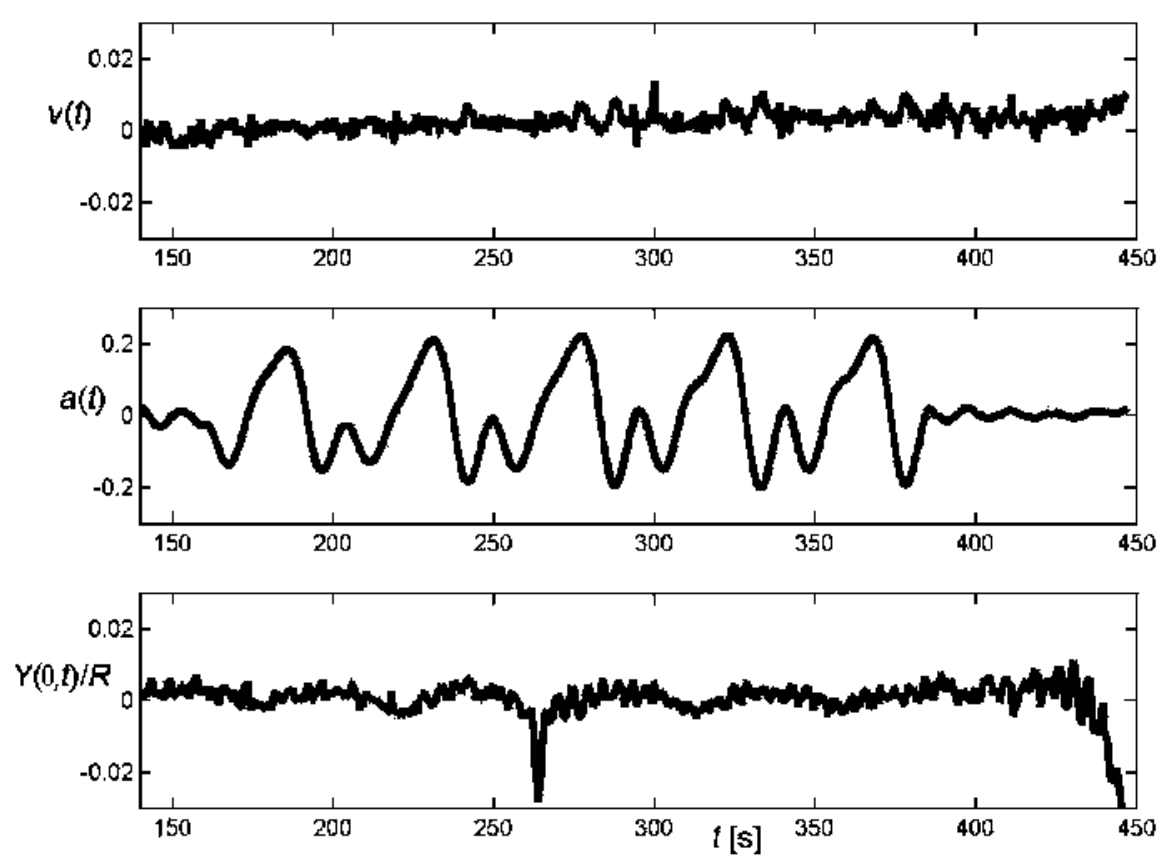

Fig. 4. Liquid bridge response obtained from whole shape image analysis, for the period of constant bridge length (i.e. the five excited cycles and the leading and trailing idle periods): non-dimensional excess volume, $v(t)=V^{*}(t) /\left(\pi \mathrm{R}^{2} L\right)-1$, non-dimensional radial departure from the cylinder, $a(t)=R(\Lambda / 2, t) / R-1$, and off-axis comb deformation at the centre, relative to the undisturbed radius, $Y(0, t) /$ $R$, vs. flight time $t$ (origin at rocket lift-off)
By getting rid of the transients in the three first cycles, a Fourier analysis of the last two excited cycles (those shown in Fig. 5) is carried out and presented in Fig. 6, where the two harmonics in the excitation $B(t)$ and the three harmonics in the response $a(t)$ clearly show up in the frequency domain (amplitude spectra $B$ and $a$, and phase spectra $\Phi_{B}$ and $\Phi_{a}$ are also shown for completeness). Notice how perfectly periodic the liquid response is in these two cycles (higher harmonics nearly zero). Frequencies have been made dimensionless with the basic frequency applied $f_{0}=1 / T$ with $T=45.2 \mathrm{~s}$.

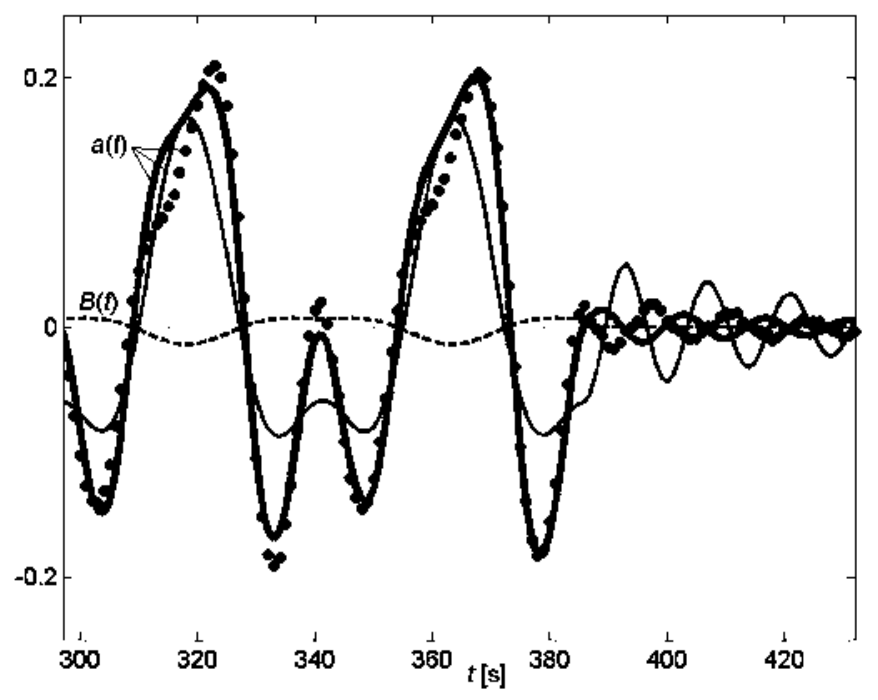

Fig. 5. Simulated time response, i.e. radial deformation at one quarter of the column length, $a(t)$, owing to the two-term load, $g(t)$, in (2). Dots correspond to experimental results, heavy solid line to the non-linear dynamic simulation, thin solid line to the linear dynamic simulation, and dashed line to applied excitation, $B(t)=\rho g(t) R^{2} / \sigma$ (non-dimensional acceleration). Only the last two excited cycles (nearly steady) and the decay are shown
The final results may be summarised as follows:

1. The non-linear dynamic simulation here performed, reasonably matches the real liquid response, contrary to the linear dynamics or any static simulation.

2. The maximum deformation amplitude in the last cycle is $a_{\max }=0.205$ (at $t=368 \mathrm{~s}$ rocket flight time, Fig. 5) that happens to be above the static stability limit for that slenderness, which is $a a_{\text {max, static }}=0.187$ according to full numerical computation. It is just the dynamic stabilisation, i.e. the fact that the force is already decreasing when the deformation is maximum (Fig. 5), which avoids the breakage of the liquid bridge-a most fortunate circumstance for the experiment. Otherwise, the liquid bridge would have already broken apart during the first transient cycles. Further simulations using (6) with a slenderness $\Lambda=2.85$ instead of the actually applied $\Lambda=2.83$ (holding all other parameters the same), already predicts the breakage of the liquid bridge, whereas a simulation with $\Lambda=2.80$ greatly decrease the response amplitude.

3. When the liquid-cell acceleration was abruptly stopped, the liquid deformation profile nearly approached zero value with zero slope (Fig. 5), i.e. the shape was a cylinder with nearly quiescent internal motion, what gave way to the abrupt decrease in amplitude deformation onwards. During the final decay, the phase difference between experiment and simulation, is because the simulation approaches this stop point slightly from above in Fig. 5, whereas the real liquid response approaches it slightly from the bottom, a minor imperfection in the simulation.

4. The final transients, although of small amplitude, fully confirm previous data for the natural frequency of this fluid configuration (Sanz 1985), namely an eigen frequency of $f_{\mathrm{e}}=0.068 \pm 0.002 \mathrm{~Hz}$, in our case, corresponding to a natural period of $T_{\mathrm{c}}=14.8 \pm 0.3 \mathrm{~s}$ and dumping time (where the amplitude reduces to a half) of some $T_{1 / 2}=35 \pm 5 \mathrm{~s}$ ). 

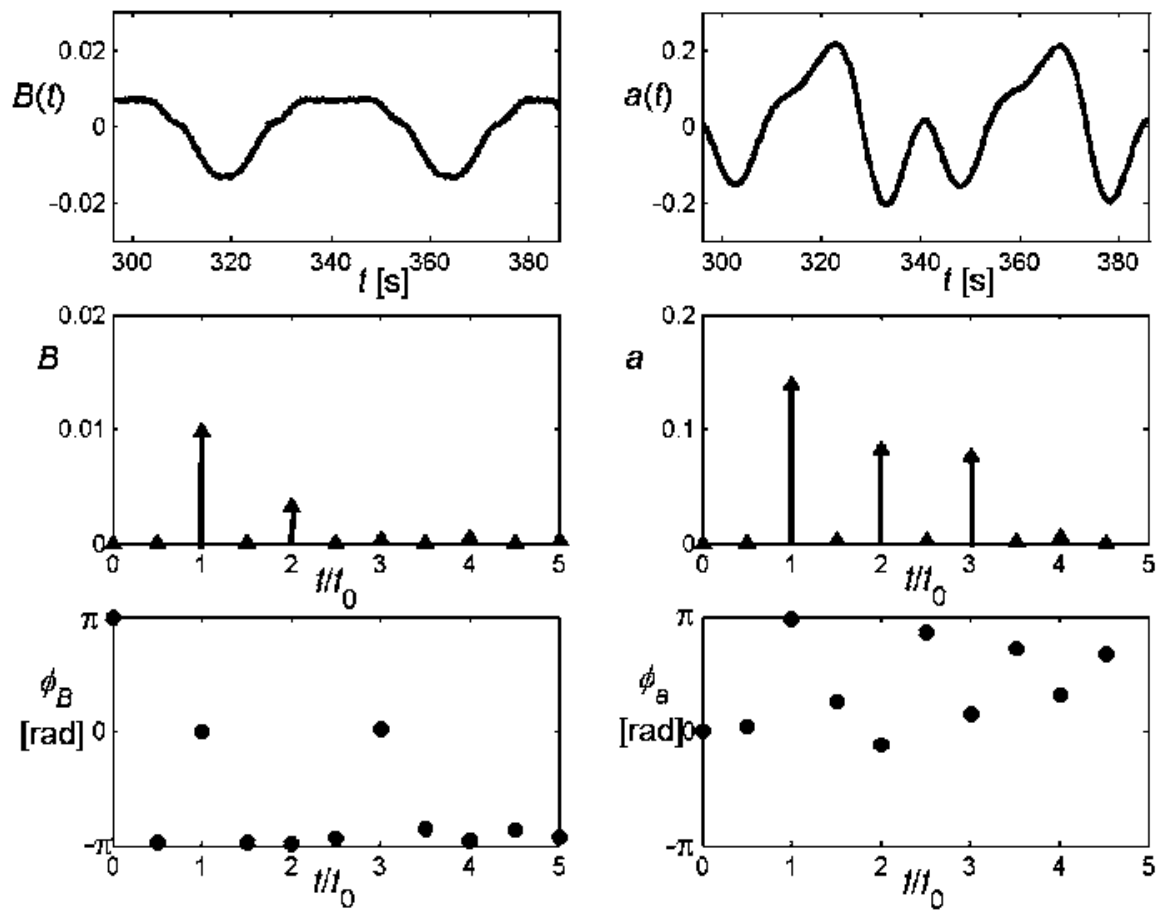

Fig. 6. Fourier analysis of input signal (dimensional acceleration excitation applied, $B(t)$ ) and output signal (maximum radial shape deformation, $a(t)=R(\Lambda)$ $2, t) / R-1)$. For both signals, the time series $(B(t)$ and $a(t))$, amplitude spectrum $(B$ and $a$ ), and phase spectrum $\left(\Phi_{B}\right.$ and $\left.\Phi_{a}\right)$ are shown. Frequencies have been made dimensionless with the basic frequency applied $f_{0}=1 / T$ with $T=45.2 \mathrm{~s}$. Only the last two excited cycles (nearly periodic) are analysed
5

\section{Conclusions}

The objective of the experiment was to check a concrete controversial quantified value: the amplitude gain of a long liquid bridge acting as a "microgravity accelerometer". The configuration used was the same as in previous trials, to minimise the parameter space. The sensitivity of the objective function to the different parameters was analysed in order to check for stability of the target and data redundancies were adopted to minimise uncertainties.

The basic aim was to verify the static response by means of a very slow sinusoidal excitation. The main result is, however, that dynamic effects were dominant. A previous analysis, including dynamic effects, tried to explain the discrepancy by introducing some unknown excitation to look for afterwards, because neither the small dynamic effect, nor the small non-linear effect could explain the results.

The present analysis has finally succeeded in explaining the discrepancy between theory and practice. The answer is that although both effects were small, their combination was not small. The two effects that reinforced each other are the following:

1. Because of the large deformation chosen to increase sensitivity, a small non-linear effect appears, less than $10 \%$ of the linear deformation, but precisely in the third power of the response amplitude. This cubic effect yields a third harmonic term in the dynamic response, which already causes the deformation to significantly deviate from the expected response (some $30 \%$ ) because the third harmonic is close to the eigen frequency of the liquid bridge.

2. The additional second harmonic excitation caused by the crank-shaft mechanism implemented, although only adding an $8 \%$ to the displacement amplitude, adds a
$33 \%$ amplitude in acceleration relative to the first harmonic. This is a sizeable departure from a simple cosine load, but without any further complications in the linear range, a perfect symmetrical response would be produced, if not for its dynamic effect.

The combination of the dynamic effects of the second harmonic excitation with the non-linear effects chosen to increase the sensitivity, finally yielded huge dynamic humps, more than $100 \%$ larger than expectations.

A simple model has been found here that perfectly simulates the real behaviour of the liquid bridge in this unique experiment. This can be applied with more confidence to make better predictions in future experiments.

Another striking fact corroborating the assertion that dynamic effects were dominant, and the validity of the present model, is the abrupt end of oscillations after the excitation ceases. The maximum measured amplitude deformation of the liquid shape is beyond the static stability limit.

\section{References}

Martínez I, Perales IM, Meseguer J (1995) Stability of long liquid columns (SL-D2- FPM-STACO). In: Sahm, PR, Keller, MH, Schiewe B (eds) Scientific results of the German Spacelab mission D-2. Wissenchaftliche Projektführung Spacelabmission D-2, Köln, pp 220-231

Martínez I, Perales JM, Meseguer J (1996) Response of a liquid bridge to an acceleration varying sinusoidally with time. Lecture Notes Phys 464:271-282

Meseguer J (1983) The breaking of axisymmetric slender liquid bridges. J Fluid Mech 130:123-151

Meseguer J, Sanz A (1985) Numerical and experimental study of the dynamics of axisymmetric slender liquid bridges. J Fluid Mech 153:83-101

Meseguer J, González MA, Alexander JID (1994) Dynamic stability of long, axisymmetric liquid bridges. Microgravity Sci Technol $7: 246-251$ 
Montanero JM (2003) Linear dynamics of axisymmetric liquid bridges. Eur J Mech B Fluids 22:167-178

Padday JF, Pétré G, Rusu CG, Gamero J, Wozniak G (1997) The shape, stability and breakage of pendant liquid bridges. J Fluid Mech 352:177-204

Rivas D, Meseguer J (1984) One-dimensional, self similar solution of the dynamics of axisymmetric slender liquid bridges. J Fluid Mech $138: 417-429$

Sanz A (1985) The influence of the outer bath in the dynamics of axisymmetric liquid bridges. J Fluid Mech 156:101-140
Sirignano WA, Mehring C (2000) Review of theory of distortion and disintegration of liquid streams. Prog Energy Combust Sci 26:609-655

Yildirim OE, Basaran OA (2001) Deformation and breakup of stretching bridges of Newtonian an shear-thinning liquids: comparison of one- and two-dimensional models. Chem Eng Sci 56:211-233

Zhang X, Padgett RS, Basaran OA (1996) Nonlinear deformation and breakup of stretching liquid bridges. J Fluid Mech 329:207-246 